Research article

Open Access

\title{
Dysfunctional interferon- $\alpha$ production by peripheral plasmacytoid dendritic cells upon Toll-like receptor-9 stimulation in patients with systemic lupus erythematosus
}

\author{
Seung-Ki Kwok ${ }^{\star}$, June-Yong Lee ${ }^{1,2 *}$, Se-Ho Park², Mi-La Cho1, So-Youn Min1, Sung-Hwan Park, \\ Ho-Youn $\mathrm{Kim}^{1}$ and Young-Gyu Cho ${ }^{1}$
}

\author{
1Department of Medicine, Division of Rheumatology, Center for Rheumatic Diseases and Rheumatism Research Center, Catholic Research Institutes \\ of Medical Sciences, Catholic University of Korea, Banpo-Dong, Seocho-Gu, Seoul, 137-701, Korea \\ ${ }^{2}$ School of Life Sciences and Biotechnology, Korea University, Anam-Dong, Seongbuk-Gu, Seoul, 136-701, Korea \\ * Contributed equally
}

Corresponding author: Ho-Youn Kim, ho0919@catholic.ac.krYoung-Gyu Cho, younggyuch0@catholic.ac.kr

Received: 9 Oct 2007 Revisions requested: 6 Nov 2007 Revisions received: 19 Feb 2008 Accepted: 6 Mar 2008 Published: 6 Mar 2008

Arthritis Research \& Therapy 2008, 10:R29 (doi:10.1186/ar2382)

This article is online at: http://arthritis-research.com/content/10/2/R29

(c) 2008 Kwok et al.; licensee BioMed Central Ltd.

This is an open access article distributed under the terms of the Creative Commons Attribution License (http://creativecommons.org/licenses/by/2.0), which permits unrestricted use, distribution, and reproduction in any medium, provided the original work is properly cited.

\begin{abstract}
Background It is well known that interferon (IFN)- $\alpha$ is important to the pathogenesis of systemic lupus erythematosus (SLE). However, several reports have indicated that the number of IFN$\alpha$ producing cells are decreased or that their function is defective in patients with SLE. We studied the function of plasmacytoid dendritic cells ( $\mathrm{pDCs}$ ) under persistent stimulation of Toll-like receptor (TLR)9 via a TLR9 ligand (CpG ODN2216) or SLE serum.

Methods The concentrations of IFN- $\alpha$ were determined in serum and culture supernatant of peripheral blood mononuclear cells (PBMCs) from SLE patients and healthy controls after stimulation with CpG ODN2216 or SLE serum. The numbers of circulating pDCs were analyzed by fluoresence-activated cell sorting analysis. pDCs were treated with CpG ODN2216 and SLE serum repeatedly, and levels of produced IFN- $\alpha$ were measured. The expression of IFN- $\alpha$ signature genes and inhibitory molecules of TLR signaling were examined in PBMCs from SLE patients and healthy control individuals.
\end{abstract}

Results Although there was no significant difference in serum concentration of IFN- $\alpha$ and number of circulating pDCs between SLE patients and healthy control individuals, the IFN- $\alpha$ producing capacity of PBMCs was significantly reduced in SLE patients. Interestingly, the degree which TLR9 ligand-induced IFN- $\alpha$ production in SLE PBMCs was inversely correlated with the SLE serum-induced production of IFN- $\alpha$ in healthy PMBCs. Because repeated stimulation pDCs with TLR9 ligands showed decreased level of IFN- $\alpha$ production, continuous TLR9 stimulation may lead to decreased production of IFN- $\alpha$ in SLE PBMCs. In addition, PBMCs isolated from SLE patients exhibited higher expression of IFN- $\alpha$ signature genes and inhibitory molecules of TLR signaling, indicating that these cells had already undergone IFN- $\alpha$ stimulation and had become desensitized to TLR signaling.

Conclusion We suggest that the persistent presence of endogenous IFN- $\alpha$ inducing factors induces TLR tolerance in pDCs of SLE patients, leading to impaired production of IFN- $\alpha$.

\section{Introduction}

Systemic lupus erythematosus (SLE) is a systemic autoimmune disease that is characterized by generation of autoantibodies against nuclear DNA and/or nuclear proteins [1]. The precise pathogenesis of SLE remains unknown, but both genetic and environmental factors are involved [2]. Over the past two decades numerous studies have suggested that interferon (IFN)- $\alpha$ may play a pathogenic role in SLE. This view is derived from the observation that IFN- $\alpha$ treatment in patients with nonautoimmune diseases, such as malignant tumors, induced anti-double-stranded DNA antibodies and occasionally resulted in the development of an SLE-like syndrome [3-7]. SLE patients exhibit ongoing IFN- $\alpha$ production, and IFN- $\alpha$ serum levels are correlated with both disease activity and

BDCA = blood dendritic cell antigen; ELISA = enzyme-linked immunosorbent assay; IFN = interferon; IL = interleukin; IRAK = IL-1 receptor-associated kinase; $\mathrm{PBMC}=$ peripheral blood mononuclear cell; PCR = polymerase chain reaction; $\mathrm{pDC}=$ plasmacytoid dendritic cell; SLE = systemic lupus erythematosus; SLEDAI = SLE Disease Activity Index; TLR = Toll-like receptor. 
severity $[8,9]$. IFN- $\alpha$ levels also correlate with anti-doublestranded DNA antibody production, complement activation, and IL-10 production, all of which are important indicators in SLE disease progression [9].

IFN- $\alpha$ plays a role in the activation, differentiation, and survival of $B$ cells, T cells, and dendritic cells. IFN- $\alpha$ is mainly produced in plasmacytoid dendritic cells (pDCs); they were originally termed natural IFN- $\alpha$ producing cells [10-12]. pDCs are key effector cells in the innate immune system because of their ability to produce large amounts of IFN- $\alpha$ in response to microbial and viral infections. Human pDCs selectively express Tolllike receptor (TLR) 7 and TLR9 within the endosomal compartment. These receptors are activated in response to viral RNA and DNA, leading to production of IFN- $\alpha[12,13]$. Recent studies have shown that DNA-containing immune complexes within SLE serum stimulate pDCs to produce IFN- $\alpha[14,15]$, which is mediated cooperatively by TLR9 and Fc $\gamma$ Rlla (CD32) [15].

Patients with SLE have reduced numbers of circulating natural IFN- $\alpha$ producing cells. The levels of IFN- $\alpha$ produced by SLE peripheral blood mononuclear cells (PBMCs) induced by SLE serum that contained an endogenous IFN- $\alpha$-inducing factor, herpes simplex virus type 1 , or the $D$ type of $C p G$ motif were lower than those produced by healthy control PBMCs, and the IFN- $\alpha$ producing capability of circulating $\mathrm{pDC}$ in SLE patients may be impaired [16-18]. These results do not fit well with the role of IFN- $\alpha$ in SLE pathogenesis described above, although they suggest that the local concentration of IFN- $\alpha$ in an affected region is important. Furthermore, there have been reports that the pDCs from SLE patients and healthy control individuals produce similar amounts of IFN- $\alpha$ on a per cell basis in response to viral infection $[17,19]$. We wished to establish definitively the IFN- $\alpha$ production capability of PBMCs from SLE patients, and we show that IFN- $\alpha$ production is dysfunctional in pDCs from such patients.

We hypothesized that the persistent presence of DNA-containing immune complexes, which stimulate TLR9, affects the function of $\mathrm{pDCs}$ resulting in their malfunction. We analyzed serum levels of IFN- $\alpha$ in SLE patients and in vitro production of IFN- $\alpha$ in isolated PBMCs subjected to artificial stimulation by CpG ODN2216, which specifically activates human TLR9 in pDCs but not in B cells [20]. We also examined the number of circulating pDCs in SLE patients, as compared with those in healthy control individuals, using specific pDC surface markers and flow cytometry. The expression of IFN- $\alpha$ signature genes (IFN- $\alpha$ responsive genes) and inhibitory molecules of the TLR signaling cascade were examined. Our findings suggest that pDCs are dysfunctional in patients with chronic SLE, which is probably due to desensitization of TLR9 as a result of over-stimulation by DNA-containing immune complexes that are present in the sera of SLE patients.

\section{Materials and methods}

Patients and control individuals

This study was approved by the Institutional Review Board of Kangnam St. Mary's Hospital (Seoul, Korea) and all participants provided informed consent. Forty-three consecutive patients (two males and 41 females; age $35 \pm 8.4$ years) who presented at the rheumatology clinic and fulfilled the revised classification criteria for SLE [21] were enrolled in the study. Twenty-six volunteers (one male and 25 females; age $38.4 \pm$ 4.3 years) were recruited to serve as healthy control individuals. Among the 43 patients, two patients were off prescription medication (in remission) and three were receiving prednisolone alone (mean dosage $12.5 \mathrm{mg} /$ day; range 5 to $20 \mathrm{mg} /$ day). The remaining 38 patients were receiving both prednisolone (mean dosage $13.3 \mathrm{mg} /$ day; range 2.5 to $125 \mathrm{mg} /$ day) and adjunctive therapies, such as hydroxychloroquine (34 patients; 200 or $400 \mathrm{mg} /$ day), azathioprine (four patients), mizoribine (four patients), mycophenolate mofetil (three patients), and cyclosporine (one patient). Disease activity was scored using the SLE Disease Activity Index (SLEDAI) [22]. Descriptive statistics and clinical data for the SLE patients are described in Table 1.

\section{Preparation of PBMCs and pDCs}

Blood samples obtained from patients and healthy control individuals were collected in heparinized tubes (BD Biosciences, San Jose, CA, USA), and PBMCs were prepared using FicollHypaque (Amersham Bioscience, Pascataway, NJ, USA) density gradient centrifugation. Cells were washed and suspended in RPMI 1640 medium (GibcoBRL, Carlsbad, CA, USA) containing penicillin $(100 \mathrm{U} / \mathrm{ml})$, streptomycin $(100 \mu \mathrm{g} /$ $\mathrm{ml}$ ), and $10 \%$ fetal bovine serum (GibcoBRL) that had been inactivated by heating to $56^{\circ} \mathrm{C}$ for 1 hour. Healthy pDCs were isolated from PBMCs using a Diamond Plasmacytoid Dendritic Cell Isolation Kit (Miltenyi Biotec, Bergisch Gladbach, Germany); pDC purity was greater than 95\%. The purified pDCs were cultured in RPMI 1640 medium containing 10\% fetal bovine serum, granulocyte-macrophage colony stimulating factor $(10 \mathrm{ng} / \mathrm{ml})$, and IL-3 $(10 \mathrm{ng} / \mathrm{ml})$.

\section{Flow cytometry}

PBMCs were incubated with human $\lg$ to block the $\mathrm{Fc}$ receptor and then incubated with anti-CD123-PE-Cy5 monoclonal antibody (Mouse $\operatorname{lgG}_{1}$; BD Pharmingen ${ }^{\mathrm{TM}}$, San Jose, CA, USA), anti-BDCA-2-fluorescein isothiocyanate, and monoclonal antibody (Mouse $\operatorname{lgG}_{1}$; Miltenyi Biotec) for 30 minutes at $4^{\circ} \mathrm{C}$; isotype control experiments were conducted in parallel. After two washes, the cells were re-suspended in phosphate-buffered saline and analyzed by flow cytometry. pDCs were identified by dual staining for both CD123 and blood dendritic cell antigen (BDCA)-2.

\section{IFN- $\alpha$ induction}

PBMCs $\left(1 \times 10^{6}\right.$ cells $)$ were stimulated using $1 \mu \mathrm{mol} / / \mathrm{CpG}$ ODN2216 (InvivoGen, San Diego, CA, USA) or 30\% serum 
Table 1

\begin{tabular}{|c|c|}
\hline Characteristic & SLE patients $(n=43)$ \\
\hline Age (years; mean [range]) & 35.0 (21 to 56$)$ \\
\hline Sex ( $n$; males/females) & $2 / 41$ \\
\hline Disease duration (years; mean [range]) & 8.5 (0.1 to 18$)$ \\
\hline SLEDAI (mean \pm SD) & $3.72 \pm 3.84$ \\
\hline Active $(n=9)^{a}$ & $9.55 \pm 4.50$ \\
\hline Inactive $(n=34)$ & $2.17 \pm 1.42$ \\
\hline Cutaneous manifestation (n [\%]) & $26(60.5)$ \\
\hline Arthritis (n [\%]) & $27(62.8)$ \\
\hline Renal manifestation (n [\%]) & $9(20.9)$ \\
\hline Cytopenia (n [\%]) & $11(25.6)$ \\
\hline Serositis (n [\%]) & $8(18.6)$ \\
\hline Prednisolone ( $n[\%]$; mean dosage $[\mathrm{mg} /$ day] $)$ & $41(95.3) ; 13.0$ \\
\hline Hydroxychloroquine (n [\%]) & $34(79.1)$ \\
\hline Azathioprine (n [\%]) & $4(9.3)$ \\
\hline Cyclosporin (n [\%]) & $1(2.3)$ \\
\hline Mizoribine (n [\%]) & $4(9.3)$ \\
\hline Mycophenolate mofetil (n [\%]) & $3(7.0)$ \\
\hline
\end{tabular}

aSystemic Lupus Erythematosus Disease Activity Index above 4. SD, standard deviation; SLE, systemic lupus erythematosus.

from SLE patients. Duplicate cultures were performed in 48well plates (NUNK, Roskilde Denmark) at a final volume of 500 $\mu \mathrm{l} /$ well. After 24 hours IFN- $\alpha$ was measured from the supernatant.

\section{Measurement of IFN- $\alpha$}

Supernatants collected from sera and cell cultures were stored at $-70^{\circ} \mathrm{C}$ until further use. The amounts of IFN- $\alpha$ in the sera and supernatants were then measured using a sensitive sandwich ELISA kit (Bender MedSystems, Vienna, Austria). A representative standard curve for the IFN- $\alpha$ ELISA is shown in Additional file 1 (Supplementary Figure 1). All measurements were performed in duplicate and averaged values were used in the data analysis.

\section{pDC stimulation with TLR9 ligand and SLE serum}

Purified pDCs $\left(2 \times 10^{4}\right.$ cells $)$ were incubated with or without the TLR9 ligand CpG ODN2216 (1 $\mu \mathrm{mol} / \mathrm{l}$; InvivoGen) or 30\% serum from SLE patients in 96-well plates (NUNK) at a final volume of $200 \mu \mathrm{l} /$ well. After 24 hours the pDCs were carefully washed with serum-free RPMI and re-treated with or without CpG ODN2216 $(1 \mu \mathrm{mol} / \mathrm{l})$ or 30\% serum from SLE patients. The supernatants were harvested after an additional 24 hours and IFN- $\alpha$ production was measured using ELISA. In the recovery assay, pDCs were treated with $1 \mu \mathrm{mol} / \mathrm{l} \mathrm{CpG}$ ODN2216 for 24 hours, washed with serum-free medium, cul- tured with serum-containing medium for 0,24 , or 48 hours, and then treated again with CpG ODN2216 (1 $\mu \mathrm{mol} / \mathrm{l})$. After 24 hours IFN- $\alpha$ production was measured from the supernatant.

\section{Cell viability assay}

Relative cell viability was measured by Quick Cell Proliferation Assay kit (BioVision, Mountain View, CA, USA). Briefly, 1/10 volume of WST-1/electrocoupling solution were added into the culture media, incubated 4 hours in 5\% carbon dioxide incubator, and measured the absorbance of the treated and untreated samples with water-soluble tetrazolium salt (WST)1 /electrocoupling solution using a microtiter plate reader at $450 \mathrm{~nm}$. Each sample was duplicated and averages of the absorbance were used in comparisons. The differences in absorbance between treated and untreated samples was shown as relative cell viability.

\section{Reverse transcription PCR}

Total RNA was extracted from isolated PBMCs or cultured cells using RNAzol B (Biotex Laboratories, Houston, TX, USA), in accordance with the manufacturer's instructions. Reverse transcription using $2 \mu \mathrm{g}$ total RNA was carried out at $42^{\circ} \mathrm{C}$ using the Superscript reverse transcription system (Takara, Shiga, Japan). PCR amplification was performed in a reaction mixture containing $2.5 \mathrm{mmol} / \mathrm{I}$ dNTPs, $2.5 \mathrm{U}$ Taq DNA 
Table 2

\begin{tabular}{ll}
\hline Sequences of primer pairs & \\
\hline Target molecules & Sequence \\
\hline GAPDH & Forward: 5'-CGA TGC TGG GCG TGA GTA C-3' \\
& Reverse: 5'-CGT TCA GCT CAG GGA TGA CC-3' \\
$\beta$-actin & Forward: 5'-GGA CTT CGA GCA AGA GAT GG-3' \\
& Reverse: 5'-TGT GTT GGC GTA CAG GTC TTT G-3' \\
TLR9 & Forward: 5'-GTG CCC CAC TTC TCC ATG-3' \\
& Reverse: 5'-GGC ACA GTC ATG ATG TTG TTG-3' \\
IFI44 & Forward: 5'-CTC GGT GGT TAG CAA TTA TTC CTC-3' \\
& Reverse: 5'-AGC CCA TAG CAT TCG TCT CAG-3' \\
IFIT1 & Forward: 5'-CTC CTT GGG TTC GTC TAC AAA TTG-3' \\
& Reverse: 5'-AGT CAG CAG CCA GTC TCA G-3' \\
PRKR & Forward: 5'-CTT CCA TCT GAC TCA GGT TT-3' \\
& Reverse: 5'-TGC TTC TGA C G GTA TGT ATT A-3' \\
MyD88s & Forward: 5'-CGG CAA CTG GAG ACA CAA G-3' \\
IRAK-M & Reverse: 5'-TCT GGA AGT CAC ATT CCT TGC-3' \\
& Forward: 5'-TTT GAA TGC AGC CAG TCT GA-3' \\
& Reverse: 5'-GCA TTG CTT ATG GAG CCA AT-3'
\end{tabular}

GAPDH, glyceraldehyde-3-phosphate dehydrogenase; IFI44, interferon-induced protein 44; IFIT1, interferon-induced protein with tetratricopeptide repeats 1; PRKR, Interferon-inducible double-stranded RNA-dependent protein kinase; TLR, Toll-like receptor.

polymerase (Takara), $0.25 \mu \mathrm{mol} / \mathrm{l}$ sense and antisense primers, and PCR buffer $\left(1.5 \mathrm{mmol} / \mathrm{l} \mathrm{MgCl}_{2}, 50 \mathrm{mmol} / \mathrm{l} \mathrm{KCl}, 10\right.$ $\mathrm{mmol} / \mathrm{l}$ Tris- $\mathrm{HCl}[\mathrm{pH} 8.3])$. Reactions were processed in a DNA thermal cycler (Perkin-Elmer Cetus, Wellesley, MA, USA). PCR products were separated on a $2.5 \%$ agarose gel and stained with ethidium bromide. The latest cycle number during which PCR products were not yet saturated was selected and used to compare treatments using Quantity One software (version 4.5.2; BioRad, Hercules, CA, USA). Results are expressed as the ratio of target PCR products to GAPDH (glyceraldehyde-3-phosphate dehydrogenase) or $\beta$-actin product. The used primer pairs are described in Table 2.

\section{Measuring immune complex levels in SLE patients' sera} To measure the serum levels of immune complexes in SLE patients, we used the CIC-C1O Circulating Immune Complexes ELISA kit (Bühlmann Laboratories AG, Schonenbuch, Switzerland). Circulating immune complexes from sera from patients with chronic SLE and control individuals were incubated with human $\mathrm{C} 1 \mathrm{q}$, which was adsorbed onto microtiter wells. After a washing step, we added alkaline phosphataseconjugated protein $A$, which binds to the $F_{c}$ region of human lgG. After an additional washing step, the enzyme substrate (paranitrophenyl-phosphate) was added, followed by a stop solution. The absorption at $405 \mathrm{~nm}$ of each sample was measured. We used 14 SLE sera, two of which were from patients with SLEDAl values of 12 and 16, and the remainder were from patients with SLEDAl values of less than 4 . The two active SLE sera exhibited $1.8 \mathrm{U}$ and $6.3 \mathrm{U}$ of immune complexes.

\section{Statistical analysis}

Differences between groups were analyzed using the MannWhitney U-test or Student's $t$-test. Correlation analyses were performed using Spearman's rank correlation test. All analyses were performed using SPSS (version 10.0; SPSS Inc., Chicago, IL, USA). Data are expressed as the mean \pm standard deviation.

\section{Results In vitro IFN- $\alpha$ production is reduced in PBMCs from SLE patients}

We used ELISA to compare serum concentrations of IFN- $\alpha$ between SLE patients and healthy control individuals (Figure 1a). Patients with active SLE (SLEDAI > 4) exhibited significantly higher levels of IFN- $\alpha$ than did patients with inactive SLE and healthy control individuals $(P=0.002$ and $P=0.007$, respectively). However, the levels of IFN- $\alpha$ in total SLE patients (active and inactive) versus healthy control individuals were not significantly different $(P=0.280)$. No correlation was observed between serum IFN- $\alpha$ levels and the clinical characteristics of SLE, such as disease duration, or medications such as steroids and hydroxychloroquine (data not shown).

Because the number of circulating pDCs is very low among total PMBCs, making it difficult to isolate this cell type from a blood sample, IFN- $\alpha$ production was measured in total PBMCs using a pDC-specific TLR9 ligand [20]. PBMCs from patients with SLE and healthy controls werestimulated in vitro for 24 hours using the TLR9 ligand CpG ODN2216. IFN- $\alpha$ production was then measured using ELISA. CpG DNA induced IFN- $\alpha$ production was markedly reduced in PBMCs from SLE patients as compared with PBMCs from healthy 

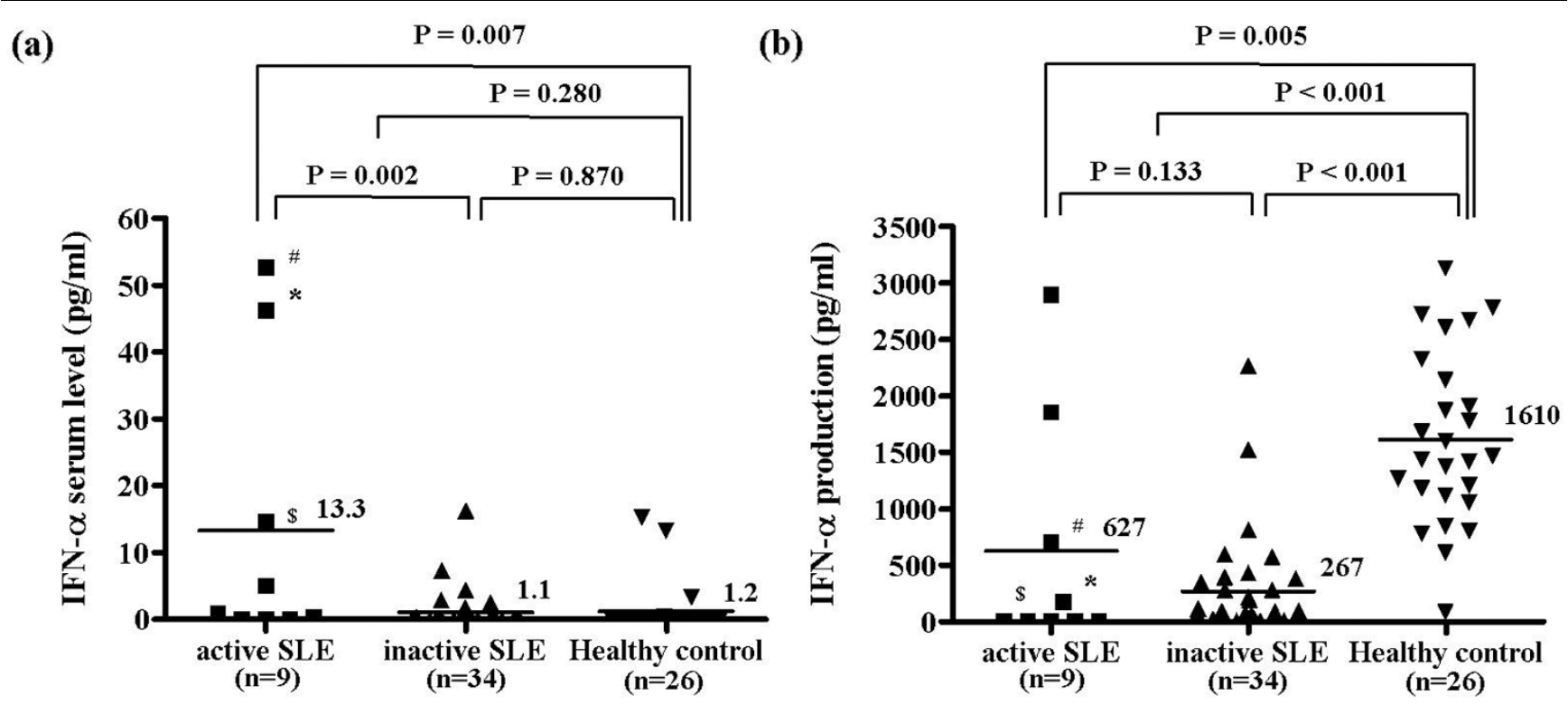

IFN- $\alpha$ serum levels and in vitro IFN- $\alpha$ production after CpG ODN2216-stimulation. (a) Interferon (IFN)- $\alpha$ serum levels were measured in blood samples collected from patients with systemic lupus erythematosus (SLE) and healthy control individuals using ELISA. Patients with a SLE Disease Activity Index above 4 were classified as having active SLE. Although serum IFN- $\alpha$ levels were higher in patients with active SLE than in those with inactive SLE and healthy control individuals, IFN- $\alpha$ levels in all SLE patients combined were not significantly different from those in healthy control individuals $(3.72 \pm 3.89 \mathrm{pg} / \mathrm{ml}$ in SLE patients [ $=43$ ] versus $1.2 \pm 3.9 \mathrm{pg} / \mathrm{ml}$ in healthy control individuals $[\mathrm{n}=26] ; P=0.280<0.001)$. (b) IFN$\alpha$ concentrations in culture supernatants were measured using ELISA after 24 hours of incubation with CpG ODN2216. IFN- $\alpha$ production in peripheral blood mononuclear cells isolated from SLE patients was significantly lower than that in cells isolated from healthy control individuals (342.46 \pm $636.82 \mathrm{pg} / \mathrm{ml}$ in SLE patients $[\mathrm{n}=43]$ versus $1,610.35 \pm 759.56 \mathrm{pg} / \mathrm{ml}$ in healthy controls $[\mathrm{n}=26] ; P<0.001)$. IFN- $\alpha$ production in peripheral blood mononuclear cells isolated from active SLE patients was also significantly lower than in cells isolated from healthy control individuals $(P=$ 0.005). The solid bars represent the mean value in each experimental group. Patients with high IFN- $\alpha$ serum levels are indicated by \#, *, and $\$$ in both experiments. Statistical significance was analyzed using Student's $t$-test.

control individuals $(342.46 \pm 636.82 \mathrm{pg} / \mathrm{ml}$ in SLE patients versus $1610.35 \pm 759.56 \mathrm{pg} / \mathrm{ml}$ in healthy control individuals; $P<0.001$; Figure 1b). In addition, IFN- $\alpha$ production was slightly higher in patients with active SLE than in those with inactive SLE, but the difference was not significant $(P=$ 0133). No correlation was observed between serum levels of IFN- $\alpha$ and $\mathrm{CpG}$-induced IFN- $\alpha$ production in vitro in patients with active SLE. Interestingly, IFN- $\alpha$ production was completely abolished in PBMCs from one-third of SLE patients. These data are almost identical to those reported previously [18]. Although CpG ODN2216 was not used in that previous work, CPG oligonucleotides, herpesviruses, and DNA-containing immune complexes are all TLR9 ligands. Our findings are in accordance with thosse of previous studies showing that PBMCs from SLE patients have reduced capacity to produce IFN- $\alpha$ in response to TLR9 stimulation $[16,17]$.

\section{Decreased IFN- $\alpha$ production in SLE patients cannot be fully explained in terms of decreased numbers of circulating pDCs}

Because pDCs are major producers of IFN- $\alpha$, decreased IFN$\alpha$ levels in SLE patients may be the result of a drop in pDC count $[16,17,23]$. To test this possibility, we stained PBMCs with anti-BDCA-2 and anti-CD123, which recognize specific surface markers for human pDCs [23]. During fluoresenceactivated cell sorting analysis, PBMCs were selected in gate $\mathrm{R} 1$ and $\mathrm{BDCA}-2^{+} / \mathrm{CD} 123^{+}$cells from gate R1 were deemed pDCs, as shown in gate R2 (Figure 2a). The percentage of pDCs among PBMCs was slightly reduced in SLE patients compared with healthy control individuals $(0.23 \pm 0.11 \%$ in SLE patients versus $0.30 \pm 0.14 \%$ in controls; $P>0.10$; Figure $2 b)$. Although this difference was not statistically significant, a decrease in pDC count may partially contribute to reduced IFN- $\alpha$ production. We observed similar results for the absolute number of circulating pDCs (data not shown). Because pDCs are a major source of IFN- $\alpha$ in human PBMCs [10-12], we calculated the relative IFN- $\alpha$ producing capacity of pDCs in vitro as follows. The amount of IFN- $\alpha$ produced upon CpG ODN2216 stimulation in vitro was divided by the absolute number of pDCs. As expected from results presented in Figures $1 b$ and $2 b$, the relative IFN- $\alpha$ producing capacity of pDCs was significantly lower in SLE patients than in control individuals $(0.2 \pm 0.18$ in SLE patients versus $1 \pm$ 0.5 in control individuals; $P<0.01$; Figure 2c). These data, and the fact that PBMCs from one-third of SLE patients did not produce IFN- $\alpha$, suggest that the observed decrease in IFN- $\alpha$ production is caused by aberrant function in SLE pDCs 
(a)

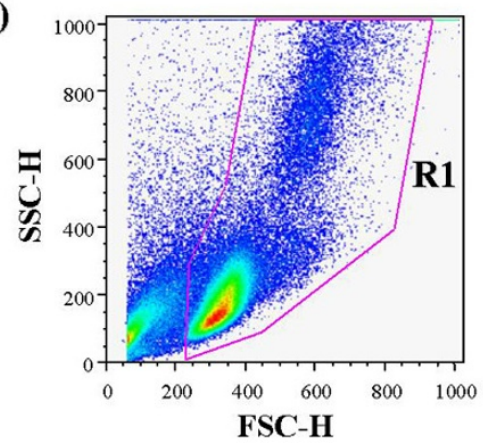

(b)

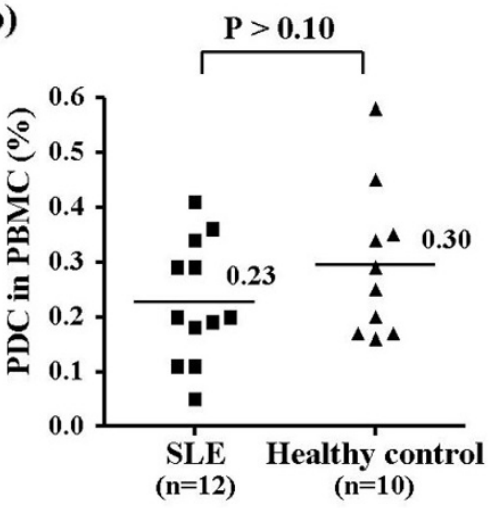

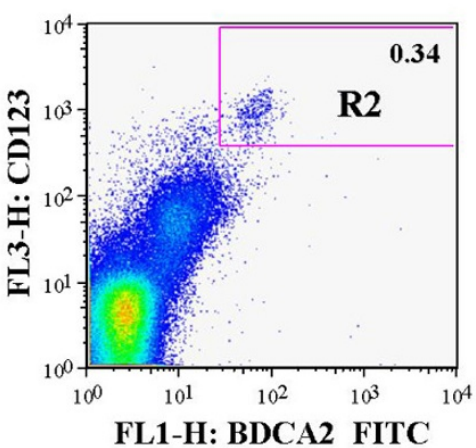

(c)

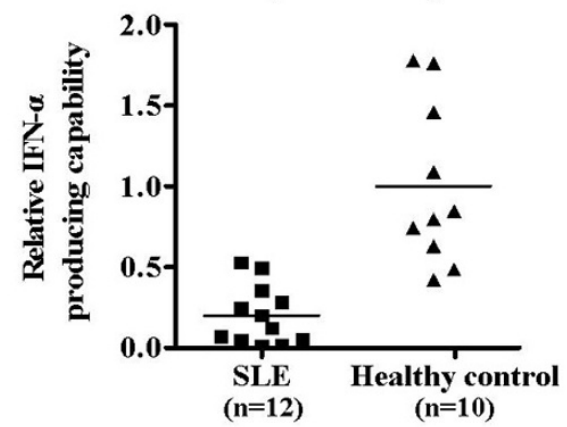

Proportion of $\mathrm{pDCs}$ and IFN- $\alpha$ production capacity. (a) A gating strategy was used to distinguish plasmacytoid dendritic cells (pDCs) from among peripheral blood mononuclear cells (PBMCs). Blood cells were analyzed using flow cytometry. Total PBMCs were gated at R1 and then analyzed for the presence of blood dendritic cell antigen (BDCA)-2 and CD123. Both BDCA-2-positive and CD123-positive cells were identified as pDCs in gate R2. (b) The proportion of pDCs among PBMCs in patients with systemic lupus erythematosus (SLE) versus healthy control individuals. The solid bars represent the mean percentage of pDCs among PBMCs $(0.23 \%$ in SLE patients versus $0.30 \%$ in controls; $P>0.10)$. Statistical significance was analyzed using the Mann-Whitney U-test. (c) Relative IFN- $\alpha$ producing capacity in SLE patients versus healthy control individuals. CpG ODN2216-induced IFN- $\alpha$ production was divided by the absolute number of pDCs. (d) Expression of Tll-like receptor (TLR) 9 mRNA. TLR-9 expression in PBMCs was measured using semiquantitative reverse transcription PCR. The expression of TLR9 is presented relative to $\beta$-actin expression. Each analysis was performed in triplicate, and the average values are indicated by a solid square for SLE patients and solid triangle for healthy control individuals. The solid bars represent the mean value for each experimental group. Statistical significance was analyzed using the Mann-Whitney U-test.

or depletion of some proportion of pDCs, and not by a decrease in total pDC count. However, there is no way to determine the proportion of pDCs in the blood.

Downregulation of TLR9 may also contribute to decreases in IFN- $\alpha$ production in SLE patients. We used semiquantitative reverse transcription $\mathrm{PCR}$ to determine the expression of TLR9 in PBMCs. Interestingly, TLR9 expression was higher in SLE PBMCs than in cells from healthy control individuals (Figure $2 \mathrm{~d}$ ). These data demonstrate that the decrease in IFN- $\alpha$ production was not caused by downregulation of TLR9 expression.

\section{CpG-induced IFN- $\alpha$ production in SLE PBMCs is inversely correlated with SLE serum induced IFN- $\alpha$ production in healthy PMBCs}

Differences in the numbers of circulating pDCs do not fully explain the difference in IFN- $\alpha$ production capability between the SLE and healthy PBMCs. Furthermore, it has been reported that TLR9 expression levels in the pDCs of SLE patients and healthy control individuals are similar [24]. We suspected that the presence of DNA-containing immune complexes, which function as TLR9 ligands, affect pDC function. In SLE patients, these immune complexes stimulate pDCs to produce IFN- $\alpha$ via TLR9 and Fc $\gamma$ Rlla (CD32) [14,15]. We incubated healthy PBMCs with serum from SLE patients and healthy control individuals, and then measured the production of IFN- $\alpha$. As expected, serum from SLE patients induced IFN$\alpha$ production to a much greater degree than did serum from healthy control individuals $(24.1 \pm 27.5 \mathrm{pg} / \mathrm{ml}$ versus $4.1 \pm$ $3.14 \mathrm{pg} / \mathrm{ml}$, respectively; $P<0.005$; Figure 3a). Paradoxically, SLE patients whose serum induces low levels of IFN- $\alpha$ production in PBMCs from healthy individuals possess PBMCs that exhibit higher IFN- $\alpha$ production when stimulated by the TLR9 ligand CpG ODN2216 (Figures 1b and 3b). Vice versa, those patients with SLE whose serum induces high lev- 
(a)

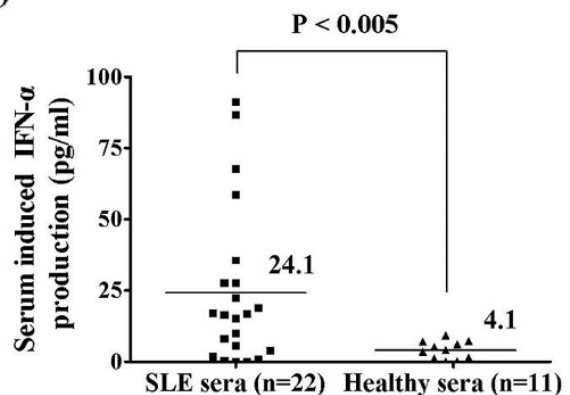

(c)

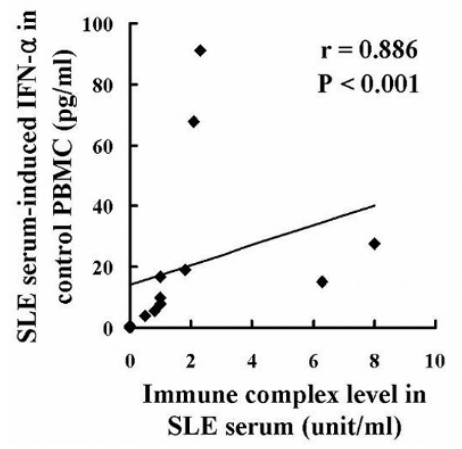

(b)

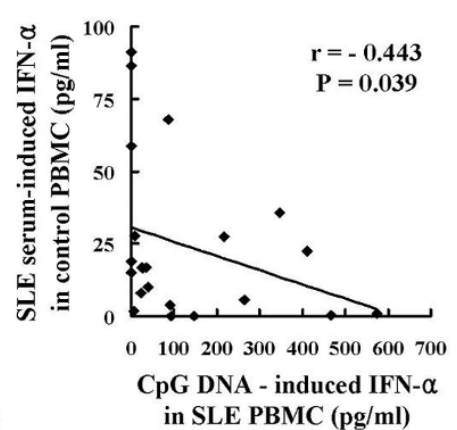

(d)

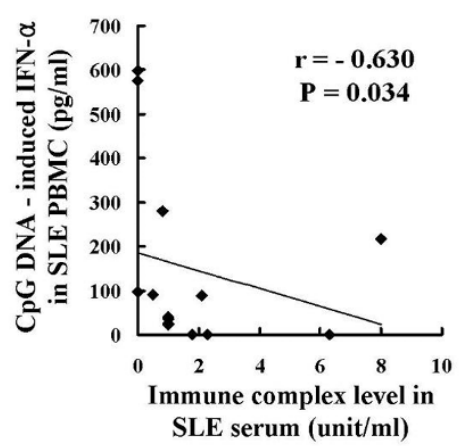

IFN- $\alpha$ production in PBMCs stimulated using serum from SLE patients or healthy control individuals. (a) Peripheral blood mononuclear cells (PBMCs), obtained from healthy individuals, were incubated with serum from patients with systemic lupus erythematosus (SLE) or healthy control individuals for 24 hours. Interferon (IFN)- $\alpha$ production was measured using ELISA. The solid bars represent the mean value for each experimental group. The experiment was performed twice using PBMCs obtained from separate healthy individuals. Data are representative of two independent experiments. (b) Inverse correlation between the amount of CpG induced IFN- $\alpha$ production in SLE PBMCs $(n=22)$ versus SLE serum induced IFN$\alpha$ in control PBMCs. (c) Positive correlation between the amounts of immune complexes versus SLE serum induced IFN- $\alpha$ in control PBMCs ( $n=$ 14). (d) Inverse correlation between the amount of CpG-induced IFN- $\alpha$ production in SLE PBMCs versus the amounts of immune complexes $(n=$ 14). Correlation analyses were performed using Spearman's rank correlation test.

els of IFN- $\alpha$ production in PBMCs from healthy individuals possess PBMCs that exhibit lower IFN- $\alpha$ production in response to CpG ODN2216. In other words, the degree of CpG-induced IFN- $\alpha$ production in SLE PBMCs correlated inversely with SLE serum induced IFN- $\alpha$ production in healthy PBMCs. Also, SLE serum induced IFN- $\alpha$ production in healthy PBMCs correlated with the amount of immune complexes in SLE serum (Figure 3c), and the degree of CpG-induced IFN$\alpha$ production in SLE PBMCs was inversely correlated with the amounts of immune complexes in matched SLE patients (Figure $3 d)$.

\section{Repeated stimulation induces TLR9 tolerance in pDCs}

Because there was an inverse correlation between IFN- $\alpha$ producing capacity in SLE PBMCs and SLE serum induced IFN$\alpha$ production in control PBMCs, we hypothesized that the persistent presence of DNA-containing immune complexes may desensitize pDCs to TLR9 stimulation. TLR tolerance, in which cells exhibit no response to subsequent challenges with the same TLR stimulus, has been reported in epithelial cells, neutrophils, macrophages, and conventional dendritic cells [2528]. To test this hypothesis, we induced TLR9 tolerance by repeatedly stimulating $\mathrm{pDCs}$ isolated from healthy individuals using CpG ODN2216 or 30\% serum derived from SLE patients. Both IL-3 and granulocyte-monocyte colony stimulating factor are essential cytokines for pDC survival and were thus included in the culture medium [29]. Repeated stimulation using CpG ODN2216 or 30\% serum from SLE patients resulted in $65 \%$ and $90 \%$ decreases in IFN- $\alpha$ production, respectively (Figure $4 \mathrm{a}$ ); cell viability was not affected (data not shown). These data indicate that repeated stimulation induces TLR9 tolerance in pDCs and are similar to previous data indicating that $\mathrm{pDC}$ s produce large amounts of IFN- $\alpha$ within the first 12 hours of activation and become refractory to subsequent stimulation [30].

TLR9 tolerance may be the result of negative feedback in the TLR signaling pathway [31]. Therefore, we examined whether TLR9 tolerance is reversible. pDC PBMCs from healthy individuals were incubated with CpG ODN2216 or 30\% SLE serum for 1 day, washed, and then re-stimulated with $\mathrm{CpG}$ ODN2216 or $30 \%$ SLE serum after a given interval $(0,24$, or 48 hours), as indicated in Figure $4 \mathrm{~b}$. IFN- $\alpha$ production was measured 24 hours after re-stimulation. IFN- $\alpha$ production decreased upon repeated stimulation at the 0 hours interval and recovered within 48 hours of the first stimulation in repeat- 


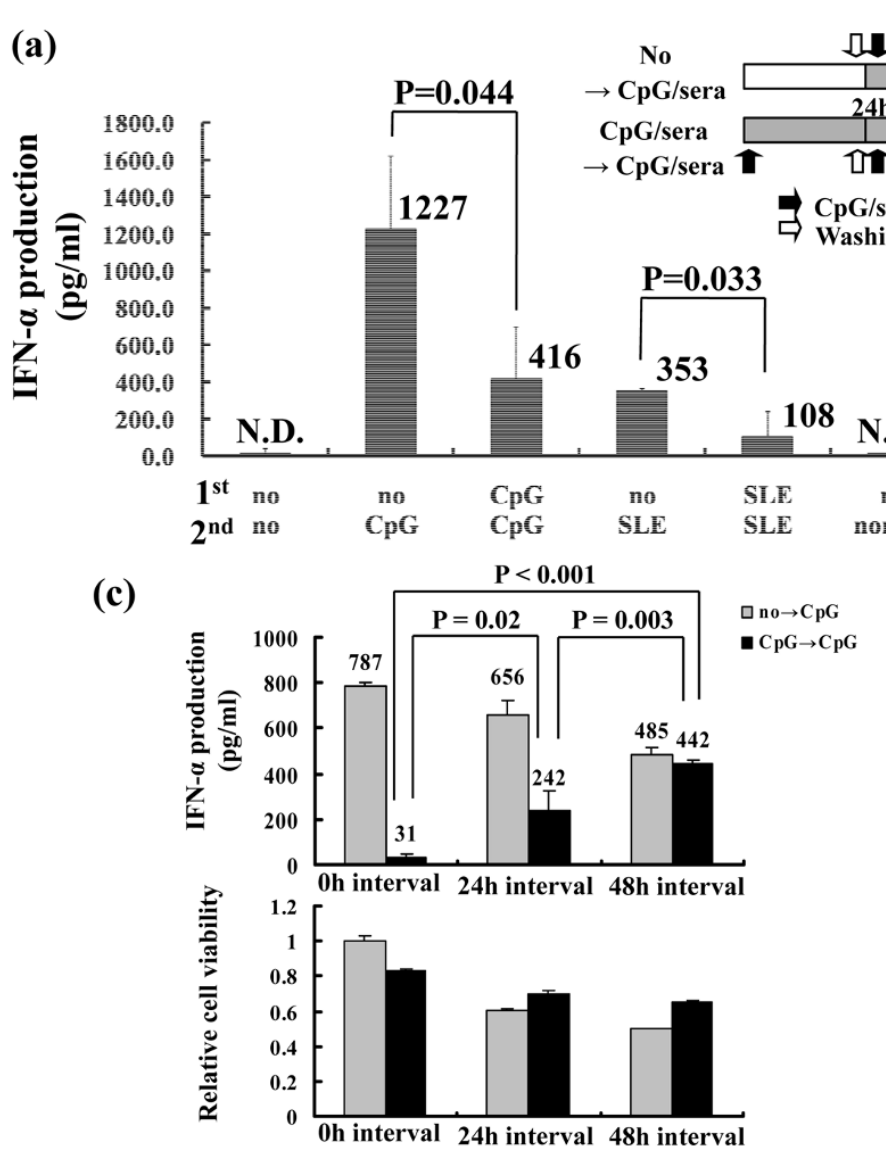

(b)

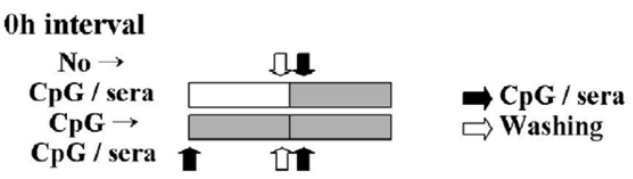

24h interval

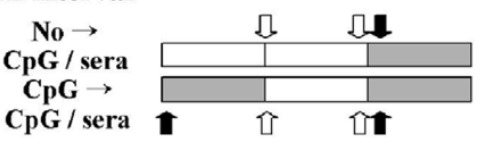

$48 \mathrm{~h}$ interval

No $\rightarrow$
CpG / sera
CpG $\rightarrow$
CpG / sera

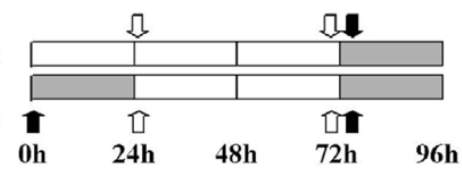

(d)

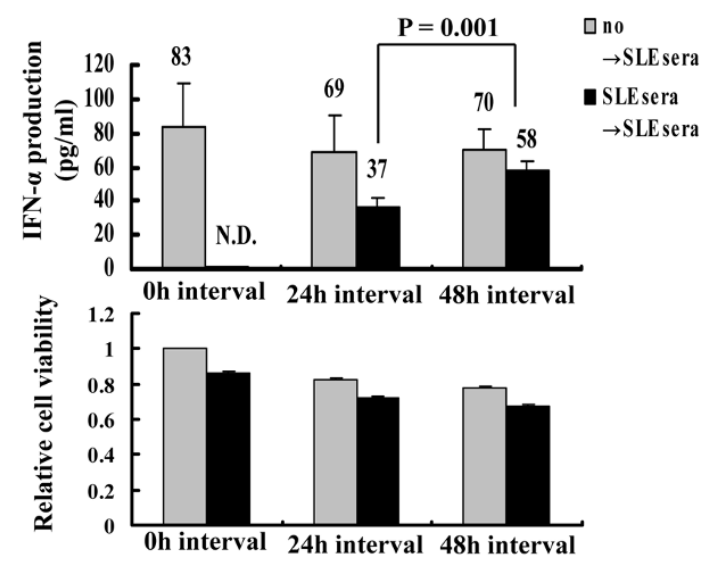

TLR9 tolerance in pDCs. (a) Repeated treatment with CpG ODN2216 and systemic lupus erythematosus (SLE) serum reduced interferon (IFN)- $\alpha$ production. Plasmacytoid dendritic cells (pDCs) were purified from peripheral blood mononuclear cells (PBMCs) of healthy individuals using Diamond Plasmacytoid Dendritic Cell Isolation Kit (Miltenyi Biotec) and $2 \times 10^{4}$ pDCs were incubated with or without CpG ODN2216 or 30\% SLE serum. After 24 hours pDCs were carefully washed in serum-free medium and then incubated again with or without CpG ODN2216 or $30 \%$ SLE serum. After 24 hours, IFN- $\alpha$ production was measured using ELISA. The experiments were performed in duplicate and three independent experiments were performed using PBMCs from different individuals. The data are presented in (Additional file 1 [Supplementary Figure 4]) and the averages of data are shown. (b) Experimental design to investigate the time-dependent recovery of Toll-like receptor (TLR) 9 sensitivity. pDCs were purified from total PBMCs of healthy individuals, and $2 \times 10^{4} \mathrm{pDCs}$ were treated with or without CpG ODN2216 or SLE sera for 1 day. The pDCs were then washed with serum-free medium and re-treated with or without CpG ODN2216 or SLE sera for 0 hours, 24 hours, and 48 hours. After 24 hours of treatment, IFN- $\alpha$ production was measured using ELISA. The white and black arrows represent washing and treatment with $\mathrm{CpG}$ ODN2216 or 30\% SLE sera, respectively. The shaded areas indicate cultures undergoing stimulation. TLR9 tolerance is reversible over time. Purified pDCs were retreated with (c) CpG ODN2216 and (d) 30\% SLE serum, as shown in panel b. IFN- $\alpha$ production and cell viability were measured 24 hours after the final stimulation. Each group was duplicated in every experiment and the values shown are the averages of duplicate samples. Three independent experiments were performed using PBMCs from different individuals. One representative case is shown here and the other data are shown in Additional file 1 (Supplementary Figure 5).

edly stimulated pDCs (Figure 4c,d). The cell viability did not differ significantly between stimulation and re-stimulation groups (lower panels of Figure 4c,d). Although we could not test the recovery of IFN- $\alpha$ production capability by $\mathrm{pDC}$ s purified from SLE patients, the PBMCs from SLE patients recovered IFN- $\alpha$ production capability over time in vitro, without further stimulation, as shown in Additional file 1 (Supplementary Figure 2). These data indicate that TLR9 tolerance is reversible over time and that continuous TLR9 stimulation is required to induce persistent nonresponsiveness in pDCs.

\section{SLE PBMCs are exposed to IFN- $\alpha$ to a greater degree}

Because the overall amounts of IFN- $\alpha$ in SLE sera were not significantly different from those in sera from healthy control individuals (Figure 1a), it was unclear whether circulating pDCs had already become TLR9 tolerant. Therefore, we used fluorescence-activated cell sorting to analyze the expression of CD80, CD86, and HLA-DR, which are markers of pDC maturation and activation. However, the number of circulating pDCs was too low to provide conclusive evidence regarding the activation of PDCs. Instead, we examined the expression of IFN- $\alpha$ signature genes in SLE PBMCs [32], including IFIT1 

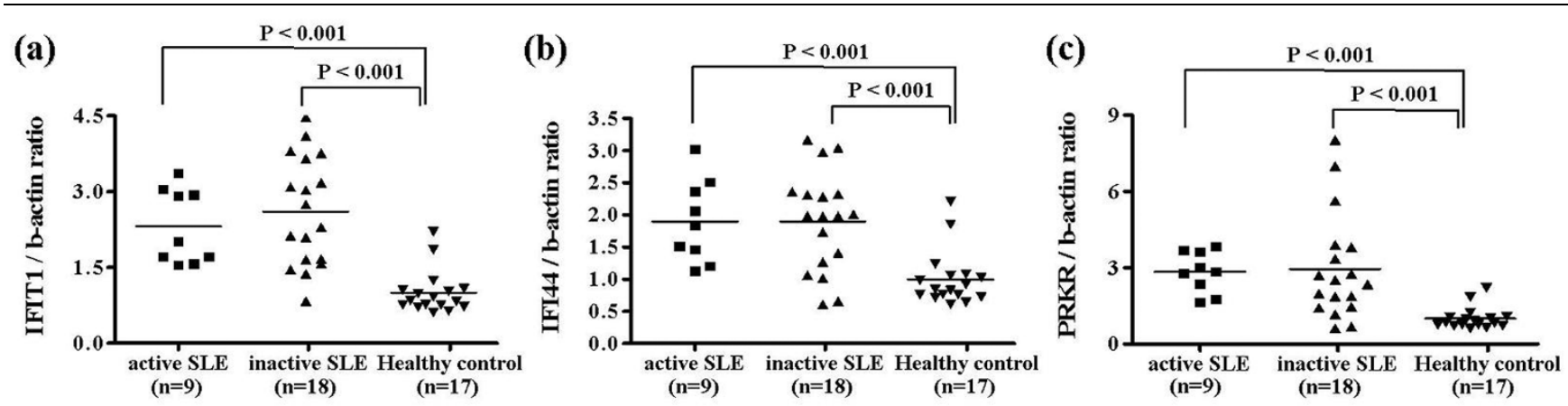

Expression of IFN- $\alpha$ signature genes. Expression of the interferon (IFN)- $\alpha$ responsive genes (a) IFI44, (b) IFIT1, and (c) PRKR was assessed using semiquantitative reverse transcription PCR. Peripheral blood mononuclear cells (PBMCs) were isolated from patients with systemic lupus erythematosus $(n=27 ; 9$ with active and 18 with inactive disease) and healthy control individuals $(n=17)$. The expression of each gene is presented relative to $\beta$-actin expression. The solid bars represent the mean value for each experimental group. Statistical significance was analyzed using Student's $t$ test.

(IFN-induced protein with tetratricopeptide repeats 1 ), IFI44 (IFN-induced protein 44), and PRKR (IFN-inducible doublestranded RNA-dependent protein kinase). The expression of all three genes was elevated in SLE PBMCs compared with healthy PBMCs (Figure $5 \mathrm{a}-\mathrm{c}$ ). These data imply that SLE PBMCs had been exposed to IFN- $\alpha$ to a greater degree, even though IFN- $\alpha$ levels did not differ between the sera of SLE patients and those of healthy control individuals.

\section{Discussion}

We demonstrated that TLR9-induced IFN- $\alpha$ production is reduced in PBMCsPMBCs from SLE patients (Figure 1 and Table 1). SLE patients exhibit ongoing IFN- $\alpha$ production, and IFN- $\alpha$ serum levels are closely correlated with SLE disease activity $[8,9]$. Although active SLE serum contains slightly increased levels of IFN- $\alpha$ as compared with inactive SLE and healthy control sera, there were no significant differences in serum levels between total or inactive SLE patients and healthy control individuals. Because some chronic SLE patients had lymphophenia (data not shown), the number of pDCs per blood unit was reduced in SLE patients, which would affect the serum levels of IFN- $\alpha$. The proportion of circulating pDCs was slightly reduced in SLE patients, and IFN$\alpha$ production was markedly impaired after in vitro stimulation with TLR9, regardless of disease activity (Figures $1 \mathrm{~b}$ and $2 \mathrm{~b}$ ). Although SLE patients exhibited a slight, nonsignificant decrease in the proportion of pDCs to total PBMCs, we cannot exclude the possibility that this decrease contributes to the decrease in TLR-induced IFN- $\alpha$ production in SLE patients, because the composition of pDC subtypes may differ between SLE patients and healthy control individuals. There is evidence that the Ly6C/Ly49Q pDC subtypes are effective producers of IFN- $\alpha$ [33], and so further investigation is required to determine the composition of pDC subtypes in SLE patients. Other studies have reported that SLE patients exhibit a reduced number of BDCA-2 expressing pDCs, and that herpes virus induced IFN- $\alpha$ production is decreased in SLE PBMCs [16]; furthermore, CpG-induced IFN- $\alpha$ secretion was significantly reduced in monocytes and dendritic cells from SLE patients [18]. However, CpG-induced IFN- $\alpha$ production was completely abolished in one-third of SLE patients, and the decrease in IFN- $\alpha$ production was more marked than the decrease in pDCs, indicating that a different mechanism is at play.

SLE patients exhibited decreased numbers of circulating pDCs (Figure 2b), which is consistent with the findings of a number of other studies [16,23,34], but they also showed increased numbers of pDCs in cutaneous lesions $[35,36]$. It has been suggested that circulating pDCs are low in SLE patients because this cell type is recruited from the blood to peripheral tissues. However, the fate of circulating pDCs after activation by DNA-containing immune complexes, which present in the blood of SLE patients, is not yet clear. Our results showed that significant numbers of $\mathrm{pDCs}$ are still present in the PBMC fraction isolated from SLE patients. Furthermore, we demonstrated that TLR-tolerant pDCs can recover over time and restore IFN- $\alpha$ production (Figure $4 c, d$ ), suggesting that $\mathrm{pDCs}$ in SLE patients are still present but inactive as a result of TLR tolerance or exhaustion.

The marked decrease or abrogation of IFN- $\alpha$ production may be explained by factors other than cell count. We noted that CpG-induced IFN- $\alpha$ production in SLE PBMCs was inversely correlated with SLE serum-stimulated cytokine production in healthy PBMCs (Figure 3b). We also found that repeated or chronic stimulation of TLR9 by appropriate ligands, such as CpG ODN 2216 or DNA-containing immune complexes, leads to TLR tolerance in pDCs. Although the mechanism of TLR tolerance has not been fully explained, it is a well known occurrence for cells that have been persistently stimulated with TLR ligands to fail to respond to re-stimulation [31,37]. One possible mechanism is inhibition of TLR signaling via dysregulation of lipopolysaccharide-induced TLR4-MyD88 com- 
plex formation and IL-1 receptor-associated kinase (IRAK)-1 activation in endotoxin-tolerant cells [38]. Another possibility is induction of genes that negatively regulate TLR signaling, such as IRAK-M and suppressor of cytokine signaling (SOCS)-1 [31,37,39].

We found an increase in the expression of IFN- $\alpha$ signature genes, indicating that SLE PBMCs have already been exposed to IFN- $\alpha$, which is mainly produced by pDCs (Figure 5). Although we did not check the expression levels of molecules that inhibit the TLR signaling cascade in pDCs from SLE patients, the SLE PBMCs showed elevated levels of IRAK-M and MyD88s compared with the healthy PBMCs (Additional file 1 [Supplementary Figure 3a,b]). Because inflammation may also increase the expression of TLR signaling molecules, we examined the expression of MyD88, which is a positive regulator of the TLR signaling pathway. MyD88 expression was also slightly elevated in SLE PBMCs (Additional file 1 [Supplementary Figure 3c]), although the ratio of MyD88s to MyD88 indicated that the negative regulator, MyD88s, was dominantly expressed in the SLE PBMCs (Additional file 1 [Supplementary Figure 3d]). Although these data do not reveal the functional status of the pDCs in SLE patients, they suggest that the expression of negative regulators of TLR signaling may be responsible for the development of TLR tolerance in the PBMCs of SLE patients. In addition, dysfunctional IFN- $\alpha$ production by SLE pDCs can be induced by other TLR ligands that are found frequently in SLE sera, such as RNA-containing immune complexes and heat shock proteins. However, our investigation was hampered by the limited number of pDCs that could be isolated from the available blood sample, and thus the exact mechanism of TLR-9 tolerance remains to be elucidated. Further investigation is required to clarify this issue.

Another possible mechanism for TLR tolerance is that SLE medications may affect the function of pDCs. Although no correlations were observed among serum IFN- $\alpha$ levels, CpGinduced IFN- $\alpha$ production in vitro, and the type and dosage of medicines taken by SLE patients (data not shown), the immuno-suppressors, such as cyclosporine and hydroxychloroquine, can affect the function of pDCs. Hydroxychloroquine, in particular, is a known inhibitor of TLR9 signaling; this drug blocks the acidification of endosomes (phagosomes), which is essential for TLR9 signaling [40]. To rule out the effect of hydroxychloroquine, pDCs from healthy individuals were pretreated with $1 \mathrm{mmol} / \mathrm{l}$ hydroxychloroquine for 24 hours, washed twice in serum-free medium, and then treated with CpG ODN2216. After 24 hours of incubation, IFN- $\alpha$ production decreased by up to $60 \%$ compared with non-pretreated pDCs (data not shown). These findings indicate that the residual amounts of hydroxychloroquine in pDCs from SLE patients may contribute to TLR tolerance. Moreover, we cannot exclude the potential influences of other medications on pDC numbers and functions. However, not all SLE patients were taking hydroxychloroquine, and the inhibition of TLR9 by residual hydroxychloroquine cannot fully explain the abrogation of IFN- $\alpha$ observed in one-third of SLE patients (Figure 1).

\section{Conclusion}

In the present study we demonstrated that circulating pDCs are desensitized to TLR9 stimulation in patients with chronic SLE. This desensitization is probably the result of persistent stimulation by DNA-containing immune complexes, which are a hallmark of SLE. In SLE patients, pDCs become tolerant to TLR9 stimulation or exhausted in terms of IFN- $\alpha$ production. These findings provide important insight into the pathogenesis of SLE and the markedly increased incidence of certain viral infections in SLE patients. In addition, our data indicate that the role of IFN- $\alpha$ is different in developing SLE and in chronic SLE.

\section{Competing interests}

The authors declare that they have no competing interests.

\section{Authors' contributions}

$\mathrm{J}-\mathrm{YL}$ and S-KK carried out the experimental work, performed the statistical analysis, and drafted the manuscript. Se-Ho P analyzed and interpreted data. M-LC and S-YM performed statistical analysis. Sung-Hwan $P$ provided patient's blood samples. $\mathrm{Y}-\mathrm{GC}$ and $\mathrm{H}-\mathrm{YK}$ designed and conceived of the study, coordinated the project, and drafted the manuscript. All authors read and approved the final manuscript.

\section{Additional files}

The following Additional files are available online:

\section{Additional file 1}

Additional file 1 is a Word file that contains

Supplementary Figures.

See http://www.biomedcentral.com/content/ supplementary/ar2382-S1.doc

\section{Acknowledgements}

This work was supported by the SRC/ERC program of MOST/KOSEF (grant R11-2002-098-08002-0).

\section{References}

1. Mok CC, Lau CS: Pathogenesis of systemic lupus erythematosus. J Clin Pathol 2003, 56:481-490.

2. Ruiz-Irastorza G, Khamashta MA, Castellino G, Hughes GR: Systemic lupus erythematosus. Lancet 2001, 357:1027-1032.

3. Ronnblom LE, Alm GV, Oberg KE: Autoimmunity after alphainterferon therapy for malignant carcinoid tumors. Ann Intern Med 1991, 115:178-183.

4. Ronnblom LE, Alm GV, Oberg KE: Possible induction of systemic lupus erythematosus by interferon-alpha treatment in a patient with a malignant carcinoid tumour. J Intern Med 1990, 227:207-210.

5. Ehrenstein MR, McSweeney E, Swane M, Worman CP, Goldstone $\mathrm{AH}$, Isenberg DA: Appearance of anti-DNA antibodies in 
patients treated with interferon-alpha. Arthritis Rheum 1993, 36:279-280.

6. Kalkner KM, Ronnblom L, Karlsson Parra AK, Bengtsson M, Olsson Y, Oberg K: Antibodies against double-stranded DNA and development of polymyositis during treatment with interferon. QJM 1998, 91:393-399.

7. Raanani $\mathrm{P}$, Ben-Bassat I: Immune-mediated complications during interferon therapy in hematological patients. Acta Haematol 2002, 107:133-144.

8. Ytterberg SR, Schnitzer TJ: Serum interferon levels in patients with systemic lupus erythematosus. Arthritis Rheum 1982, 25:401-406.

9. Bengtsson AA, Sturfelt G, Truedsson L, Blomberg J, Alm G, Vallin $H$, Ronnblom $L$ : Activation of type I interferon system in systemic lupus erythematosus correlates with disease activity but not with antiretroviral antibodies. Lupus 2000, 9:664-671.

10. Cella M, Jarrossay D, Facchetti F, Alebardi O, Nakajima H, Lanzavecchia $A$, Colonna $M$ : Plasmacytoid monocytes migrate to inflamed lymph nodes and produce large amounts of type I interferon. Nat Med 1999, 5:919-923.

11. Siegal FP, Kadowaki N, Shodell M, Fitzgerald-Bocarsly PA, Shah $\mathrm{K}, \mathrm{Ho}$ S, Antonenko S, Liu YJ: The nature of the principal type 1 interferon-producing cells in human blood. Science 1999, 284:1835-1837.

12. Colonna M, Trinchieri G, Liu YJ: Plasmacytoid dendritic cells in immunity. Nat Immunol 2004, 5:1219-1226.

13. Ito T, Wang YH, Liu YJ: Plasmacytoid dendritic cell precursors/ type I interferon-producing cells sense viral infection by Tolllike receptor (TLR) 7 and TLR9. Springer Semin Immunopathol 2005, 26:221-229.

14. Lovgren T, Eloranta ML, Bave U, Alm GV, Ronnblom L: Induction of interferon-alpha production in plasmacytoid dendritic cells by immune complexes containing nucleic acid released by necrotic or late apoptotic cells and lupus IgG. Arthritis Rheum 2004, 50:1861-1872

15. Means TK, Latz E, Hayashi F, Murali MR, Golenbock DT, Luster AD: Human lupus autoantibody-DNA complexes activate DCs through cooperation of CD32 and TLR9. J Clin Invest 2005, 115:407-417.

16. Blomberg S, Eloranta ML, Magnusson M, Alm GV Ronnblom L: Expression of the markers BDCA-2 and BDCA-4 and production of interferon-alpha by plasmacytoid dendritic cells in systemic lupus erythematosus. Arthritis Rheum 2003, 48:2524-2532

17. Cederblad B, Blomberg S, Vallin H, Perers A, Alm GV, Ronnblom $\mathrm{L}:$ Patients with systemic lupus erythematosus have reduced numbers of circulating natural interferon-alpha-producing cells. J Autoimmun 1998, 11:465-470.

18. Zeuner RA, Klinman DM, Illei G, Yarboro C, Ishii KJ, Gursel M, Verthelyi $D$ : Response of peripheral blood mononuclear cells from lupus patients to stimulation by $\mathrm{CpG}$ oligodeoxynucleotides. Rheumatology (Oxford) 2003, 42:563-569.

19. Blanco P, Palucka AK, Gill M, Pascual V, Banchereau J: Induction of dendritic cell differentiation by IFN-alpha in systemic lupus erythematosus. Science 2001, 294:1540-1543.

20. Krug A, Rothenfusser S, Hornung V, Jahrsdorfer B, Blackwell S, Ballas ZK, Endres S, Krieg AM, Hartmann G: Identification of $\mathrm{CpG}$ oligonucleotide sequences with high induction of IFNalpha/beta in plasmacytoid dendritic cells. Eur J Immunol 2001, 31:2154-2163.

21. Hochberg MC: Updating the American College of Rheumatology revised criteria for the classification of systemic lupus erythematosus. Arthritis Rheum 1997, 40:1725.

22. Bombardier C, Gladman DD, Urowitz MB, Caron D, Chang $\mathrm{CH}$ : Derivation of the SLEDAI. A disease activity index for lupus patients. The Committee on Prognosis Studies in SLE. Arthritis Rheum 1992, 35:630-640.

23. Migita K, Miyashita T, Maeda Y, Kimura H, Nakamura M, Yatsuhashi $\mathrm{H}$, Ishibashi H, Eguchi K: Reduced blood BDCA-2+ (Iymphoid) and $\mathrm{CD}_{11 \mathrm{c}^{+}}$(myeloid) dendritic cells in systemic lupus erythematosus. Clin Exp Immuno/ 2005, 142:84-91.

24. Migita K, Miyashita T, Maeda Y, Nakamura M, Yatsuhashi $H$, Kimura $\mathrm{H}$, Ishibashi $\mathrm{H}$, Eguchi $\mathrm{K}$ : Toll-like receptor expression in lupus peripheral blood mononuclear cells. J Rheumatol 2007, 34:493-500.
25. Dalpke AH, Lehner MD, Hartung T, Heeg K: Differential effects of CpG-DNA in Toll-like receptor-2/-4/-9 tolerance and crosstolerance. Immunology 2005, 116:203-212.

26. Lotz M, Gutle D, Walther S, Menard S, Bogdan C, Hornef MW Postnatal acquisition of endotoxin tolerance in intestinal epithelial cells. J Exp Med 2006, 203:973-984.

27. Parker LC, Jones EC, Prince LR, Dower SK, Whyte MK, Sabroe I: Endotoxin tolerance induces selective alterations in neutrophil function. J Leukoc Biol 2005, 78:1301-1305.

28. Perry JA, Olver CS, Burnett RC, Avery AC: Cutting edge: the acquisition of TLR tolerance during malaria infection impacts T cell activation. J Immuno/ 2005, 174:5921-5925.

29. Grouard G, Rissoan MC, Filgueira L, Durand I, Banchereau J, Liu YJ: The enigmatic plasmacytoid T cells develop into dendritic cells with interleukin (IL)-3 and CD40-ligand. J Exp Med 1997, 185:1101-1111.

30. Ito T, Kanzler H, Duramad O, Cao W, Liu YJ: Specialization, kinetics, and repertoire of type 1 interferon responses by human plasmacytoid predendritic cells. Blood 2006, 107:2423-2431.

31. Fan H, Cook JA: Molecular mechanisms of endotoxin tolerance. $J$ Endotoxin Res 2004, 10:71-84.

32. Hua J, Kirou K, Lee C, Crow MK: Functional assay of type I interferon in systemic lupus erythematosus plasma and association with anti-RNA binding protein autoantibodies. Arthritis Rheum 2006, 54:1906-1916.

33. Vremec D, O'Keeffe M, Hochrein H, Fuchsberger M, Caminschi I, Lahoud M, Shortman K: Production of interferons by dendritic cells, plasmacytoid cells, natural killer cells, and interferonproducing killer dendritic cells. Blood 2007, 109:1165-1173.

34. Vallin H, Blomberg S, Alm GV, Cederblad B, Ronnblom L: Patients with systemic lupus erythematosus (SLE) have a circulating inducer of interferon-alpha (IFN-alpha) production acting on leucocytes resembling immature dendritic cells. Clin Exp Immunol 1999, 115:196-202.

35. Blomberg S, Eloranta ML, Cederblad B, Nordlin K, Alm GV, Ronnblom L: Presence of cutaneous interferon-alpha producing cells in patients with systemic lupus erythematosus. Lupus 2001, 10:484-490.

36. Farkas L, Beiske K, Lund-Johansen F, Brandtzaeg P, Jahnsen FL: Plasmacytoid dendritic cells (natural interferon- alpha/betaproducing cells) accumulate in cutaneous lupus erythematosus lesions. Am J Pathol 2001, 159:237-243.

37. Akira S, Takeda K: Functions of toll-like receptors: lessons from KO mice. C R Bio/ 2004, 327:581-589.

38. Medvedev AE, Lentschat A, Wahl LM, Golenbock DT, Vogel SN: Dysregulation of LPS-induced Toll-like receptor 4-MyD88 complex formation and IL-1 receptor-associated kinase 1 activation in endotoxin-tolerant cells. J Immunol 2002, 169:5209-5216.

39. Escoll P, del Fresno C, Garcia L, Valles G, Lendinez MJ, Arnalich F, Lopez-Collazo E: Rapid up-regulation of IRAK-M expression following a second endotoxin challenge in human monocytes and in monocytes isolated from septic patients. Biochem Biophys Res Commun 2003, 311:465-472.

40. Rutz M, Metzger J, Gellert T, Luppa P, Lipford GB, Wagner H, Bauer S: Toll-like receptor 9 binds single-stranded CpG-DNA in a sequence- and $\mathrm{pH}$-dependent manner. Eur J Immunol 2004, 34:2541-2550. 